\title{
Game-Theoretic Market-Driven Smart Home Scheduling Considering Energy Balancing
}

\author{
Yang Liu, Shiyan Hu, Senior Member, IEEE, Han Huang, Senior Member, IEEE, \\ Rajiv Ranjan, Member, IEEE, Albert Y. Zomaya, Fellow, IEEE, and Lizhe Wang, Senior Member, IEEE
}

\begin{abstract}
In a smart community infrastructure that consists of multiple smart homes, smart controllers schedule various home appliances to balance energy consumption and reduce electricity bills of customers. In this paper, the impact of the smart home scheduling to the electricity market is analyzed with a new smart-home-aware bi-level market model. In this model, the customers schedule home appliances for bill reduction at the community level, whereas aggregators minimize the energy purchasing expense from utilities at the market level, both of which consider the smart home scheduling impacts. A game-theoretic algorithm is proposed to solve this formulation that handles the bidirectional influence between both levels. Comparing with the electricity market without smart home scheduling, our proposed infrastructure balances the energy load through reducing the peak-to-average ratio by up to $35.9 \%$, whereas the average customer bill is reduced by up to $34.3 \%$.
\end{abstract}

Index Terms-Dynamic pricing, electricity market, energy balancing, game theory, smart home scheduling.

\section{INTRODUCTION}

$\mathbf{S}$ MART home scheduling provides management over the home appliances in the smart grid infrastructure. All the home appliances in a smart home are connected to a smart home scheduler and the power line, which is further connected to the local distribution network, as shown in Fig. 1. The data depicted in Fig. 2 indicate that the energy price at different time slots would be significantly different even in a single day. For this reason, the smart home scheduler controls home appliances and operates them at the time slots when energy is not expensive, thus reducing the monetary cost. This enables the customers

Manuscript received November 18, 2014; revised January 26, 2015; accepted March 5, 2015. The work of R. Ranjan was supported by the AustraliaIndia Strategic Research Fund entitled "Innovative Solutions for Big Data and Disaster Management Applications on Clouds" under Grant AISRF08140 through the Department of Industry, Australia. (Corresponding authors: Shihan Hu and Lizhe Wang.)

Y. Liu and S. Hu are with the Department of Electrical and Computer Engineering, Michigan Technological University, Houghton, MI 49931 USA (e-mail: yliu18@mtu.edu; shiyan@mtu.edu).

H. Huang is with the State Grid Energy Research Institute, State Grid Corporation of China, Beijing 100031, China (e-mail: huanghan@sgeri.sgcc.com.cn).

R. Ranjan is with the Commonwealth Scientific and Industrial Research Organisation (CSIRO), Canberra, A.C.T. 2600, Australia (e-mail: rrajv.ranjan@ csiro.au).

A. Y. Zomaya is with the School of Information Technologies, The University of Sydney, Darlington, N.S.W. 2008, Australia (e-mail: albert.zomaya@ sydney.edu.au).

L. Wang is with the School of Computer Science, China University of Geoscience, Wuhan, 430074, China, and also with the Institute of Remote Sensing and Digital Earth, Chinese Academy of Sciences, Beijing 100094, China (e-mail: lizhe.wang@computer.org).

Digital Object Identifier 10.1109/JSYST.2015.2418032

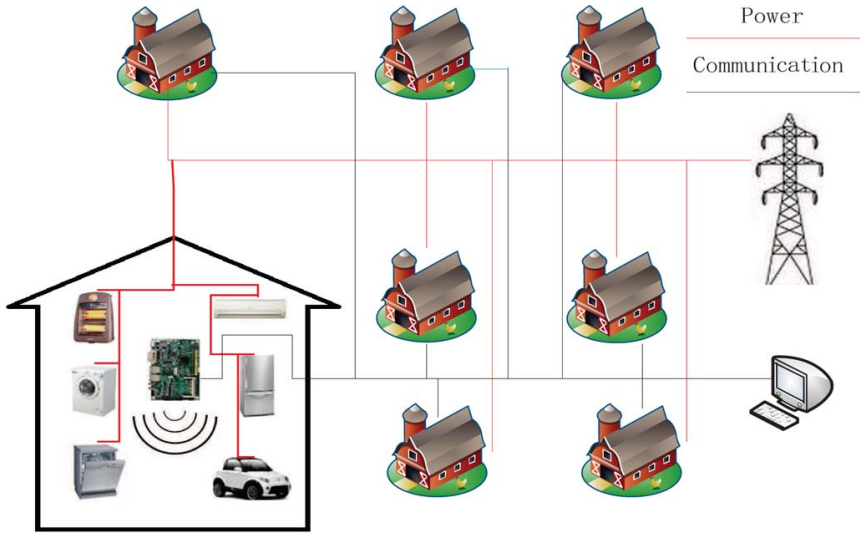

Fig. 1. In a smart home, the home appliances are connected to the smart home scheduler by communication network and connected to the distribution system by power line.

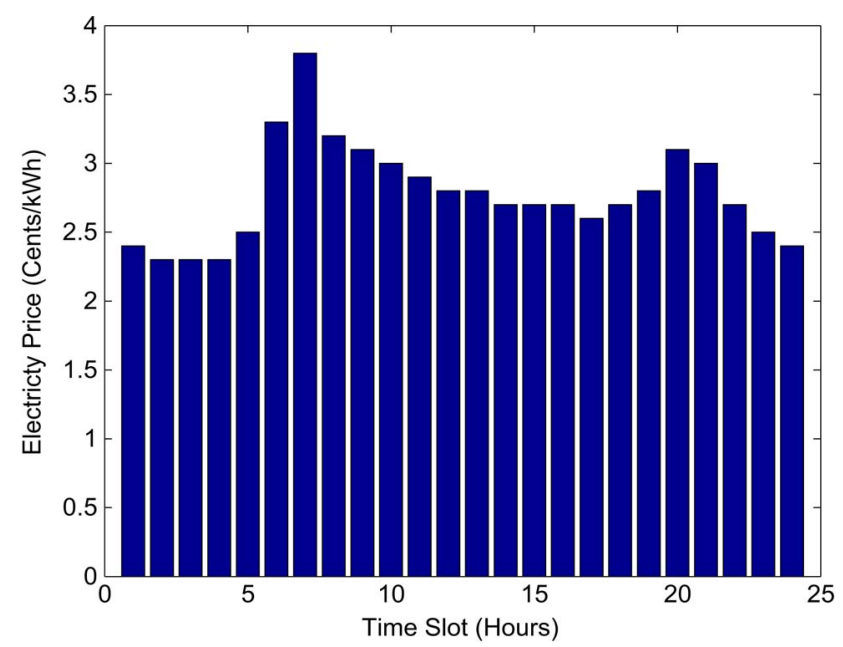

Fig. 2. Dynamic energy price provided by Ameren [4].

to shift heavy consumption load from peak price time slots to nonpeak price time slots [1]. With an appropriately designed pricing scheme, both the monetary costs of the customers and the peak-to-average ratio (PAR) of energy demand could be significantly reduced [2]. This implicitly helps balance the energy generation and reduces the generation capacity, which lessens the need of large-scale power plants, thus saving a large amount of construction cost. As shown from the U.S. Energy Information Administration, the total capital cost of building an advanced pulverized coal plant with a nominal capacity of $650 \mathrm{MW}$ is about 2.1 billion U.S. dollars [3]. Utilizing smart home scheduling techniques could largely reduce peak 
energy demand, leading to the significant investment savings in building power generation units.

Research on smart home scheduling focuses on both single customer smart home and multiple customer smart homes. In terms of single customer smart home scheduling, Chen et al. formulated a linear programming problem for smart home scheduling considering the uncertainty of energy consumption in [1]. In [5], Kim and Poor proposed a Markov chain model of the scheduling problem and developed the backtrack algorithm to solve it based on a decision threshold. In [6], a dynamic programming algorithm was proposed to schedule home appliances for a single customer considering multiple power levels. In [7], a mixed-integer programming method was used to handle the constraints such as uninterruptable setting and sequential operations in the smart home scheduling problem. In [8], $L_{1}$ regulation was deployed to transform a mixed-integer problem into a convex programming problem in order to find the solution of smart home scheduling more efficiently. All of these works focus on smart home scheduling for a single customer. However, in a community, there are multiple customers, and all the customers compete to use energy in nonpeak price time slots, which could result in the accumulation of energy load in these time slots. For this reason, interactions among multiple customers need to be considered.

Most of the existing multiple customer smart home scheduling techniques are based on game theory, which include [2], [9], and [10]. In addition, Ibars et al. formulated the distributed load management problem as a congestion game and proposed a dynamic pricing strategy to discourage the energy consumption at peak hours in [11]. In [12], the vehicle-to-aggregator game was modeled to regulate the frequency in the power grid as a potential service in the future vehicle-to-grid market. In [13] and [14], pricing strategies were deployed by local aggregators to control the energy load. Since the residential energy load is shifted by smart home scheduling, an impact propagates to the electricity market, which is never studied in the existing literatures.

On the other hand, electricity market modeling is a wellstudied research topic. In [15], the electricity market in Northern Europe was modeled considering various generation resources and transmission protocols. In [16], the agent-based simulation method was used via the Electricity Market Complex Adaptive System to investigate the influence of price probing strategies on the electricity price and generation profit. In [17], a single buyer market model and a pool market model are compared in terms of generation revenue. In [18], different game-theory-based market models were compared in terms of their market performance. The existing works on electricity market modeling use statistical data rather than the actual behavior on energy consumption of customers [19]. However, there is no guarantee that the statistically estimated energy consumption of customers can be actually achieved during power system operation. It means that the feasibility of these models cannot be guaranteed.

It is worth noting that demand-side management (DSM) and smart home scheduling are different. As a top-down energy load management technique, the traditional DSM, or precisely direct load control (DLC), intends to balance the energy load
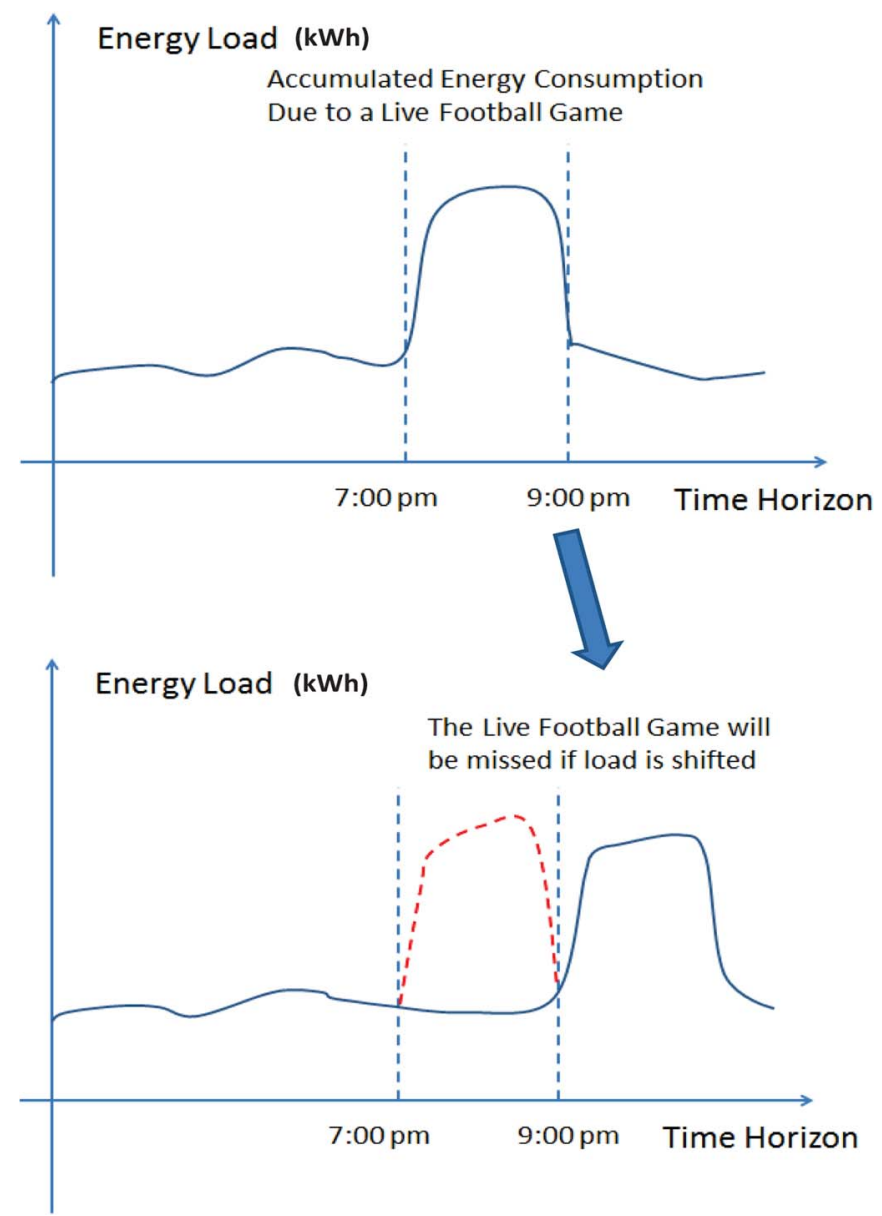

Fig. 3. Suppose that a peak energy load is created between 7:00 P.M. and 9:00 P.M. due to a live TV football game. It cannot be shifted since it will be missed otherwise.

through enabling the utilities to control the energy consumption of the customers. However, as shown in Fig. 3, if most home appliances can only be operated during a specific time period, energy load will accumulate to peak there anyway, and the majority of the energy consumption cannot be shifted at all. It means that the strategies of market to schedule the energy consumption are not applicable without considering the specific requirements from customers. In contrast to DLC, smart home scheduling provides a more desirable solution of this problem in the bottom-up fashion. It encourages the customers to allocate the energy consumption evenly over the time horizon through dynamic pricing, which is widely accepted today. Therefore, an electricity market model considering smart home is crucial in analyzing the modern power system.

Energy scheduling is influenced by the market pricing strategy, which, in turn, affects the electricity price. This forms a feedback loop and imposes more challenge to the modeling of electricity market. The bidirectional interaction between utilities and end users need to be considered. Due to such a feature of the electricity market, a feedback control system is widely deployed to model the interaction between the suppliers and the consumers. In [20], Voice et al. proposed a feedbackloop-based strategy to manage the microstorage system, where the suppliers use adaptive pricing to interact with the storage 


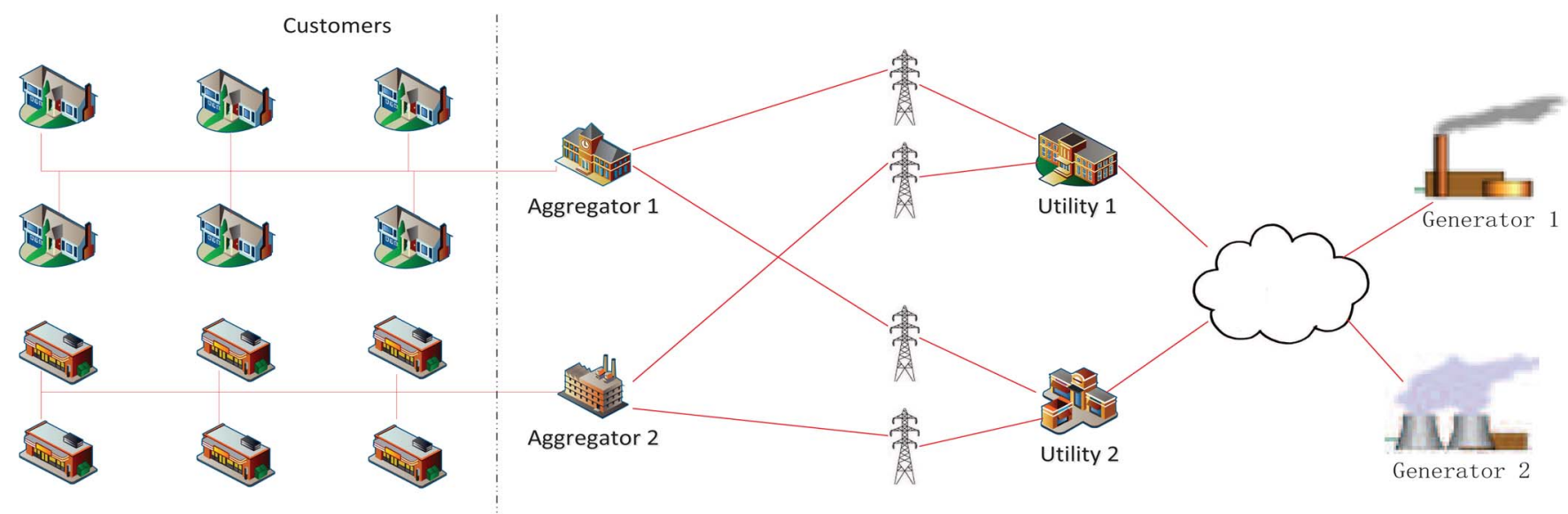

Fig. 4. Simplified power system model consisting of generators, utilities aggregators, and customers.

agents. In [13], Kishore and Snyder formulated a Stackelberg game to model the competition between the customers and the retailers for energy load controlling. In [21], Ramchurn et al. designed the control mechanism for green energy considering the feedback between the autonomous energy storage system and the green energy supplier operation. In [22], Kok et al. compared several existing techniques to use the end-user feedback in smart grids, including automated decentralized control of distributed generation and demand response and control for grid stability and islanding operation. In contrast to these works, our proposed electricity market model incorporates game theory into the feedback loop to model the competition at both the market and community levels.

To the best of our knowledge, this paper presents the first study of the impact of smart home scheduling to electricity market. A bi-level model is proposed, which takes into consideration smart home scheduling and competitions among customers at the community level and among multiple communities at the market level. Our contributions are listed as follows.

1) This paper is the first work addressing the impact of smart home scheduling to the electricity market.

2) A bi-level game is formulated, where each customer competes to minimize its individual monetary cost in the community-level game and each aggregator competes to minimize the monetary cost of its community in the market-level game. The bidirectional interaction between the market level and the community level is also modeled.

3) To solve the game formulation, an energy demand partition-based market purchasing algorithm is proposed, and a top-level algorithm to modulate the market purchasing and smart home scheduling is also proposed.

4) Simulations are conducted using a test case consisting of two utilities and five communities, where each community contains 400 customers. The simulation results demonstrate that comparing with the technique without smart home scheduling, the average monetary cost of each customer is reduced by $31.8 \%$ and $34.3 \%$ for weekdays and weekends, respectively. The PAR is reduced by $35.9 \%$ and $24.9 \%$ for weekdays and weekends, respectively. It is also observed that the generation is balanced over the time horizon. The capacity of a power generator is reduced by $29.8 \%$ and $24.9 \%$ for weekdays and weekends, respectively.

This paper is organized as follows. In Section II, the preliminaries of the smart home scheduling infrastructure are presented. In Section III, the proposed electricity market models and corresponding algorithms are described. In Section IV, the results of simulations are presented and analyzed. A summary of work is given in Section V.

\section{PRELIMINARY}

A power system consisting of customers, aggregators, utilities, and generators is depicted in Fig. 4. Let the community level refer to the interaction between customers and aggregators, and let the market level refer to the interactions among aggregators, utilities, and generators.

\section{A. Community Level}

1) Aggregators: The aggregator provides management over the community. In the community level, the aggregator receives the electricity price from the market and delivers it to the customers. It also collects the energy demand request of each customer and broadcasts it to all others.

2) Customers: The customers are equipped with smart home schedulers, which also exchange information with the aggregator. The mechanisms of the smart home scheduler are provided in Section III. The home appliances of the customers can generally be divided into three categories as follows. 1) The first category contains home appliances with multiple power levels that are automatically schedulable such as washing machines, cloth driers, plug-in electric vehicles (PHEVs), and dish washers. For this type of home appliances, power levels could be adjusted subject to the constraints on start time and deadline, in which start time is the time point that a home appliances can start to work and deadline is the time point that a home appliance has to finish working. 2) The second category contains manually controlled home appliances such as TV sets and computers. 3) The third category contains home appliances turned on all day long such as refrigerators. The smart home scheduling technique controls these home appliances with different strategies according to their features. 


\section{B. Market Level}

Here, the market activities are discussed, including the trading between utilities and generators and the trading between utilities and aggregators. In terms of the trading between utilities and generators, two types of markets, including forward market and wholesale market [23], are modeled. In different types of markets, the utilities and generators have a different trading price, which is also a reference for the utilities to set the selling price to the aggregators.

1) Utilities Versus Generators: Utilities make profits through purchasing electricity energy from generators and selling it to aggregators. In the forward market, each utility has a contract with its local generator, according to which the utility purchases a certain amount of energy from the local generator with a fixed total price. The energy amount is defined as the forward limit. If the requested amount exceeds the forward limit, the utility purchases the rest of the energy demand in the wholesale market, where the price is much higher. In that situation, the utility can choose to purchase from either the local generator or remote generators according to the locational marginal price (LMP) [24].

2) Utilities Versus Aggregators: In the trading between utilities and aggregators, an aggregator purchases energy from utilities to satisfy the total energy demand of the community. Among all the available utilities, the aggregator chooses the ones with the lowest prices to minimize the total monetary cost of the community. The specific pricing strategy is presented in Section III.

\section{Proposed Model And Algorithms}

Here, the analytical models in community and market levels are proposed. In terms of smart home scheduling and market trading, two optimization problems are formulated, respectively, based on which the bi-level game is developed. A bi-level algorithm consisting of a smart home scheduling algorithm and a market purchasing algorithm is proposed to solve the optimization problems. Throughout this paper, the index of communities is denoted by $n \in\{1,2, \ldots, N\}$, the index of utilities is denoted by $m \in\{1,2, \ldots, M\}$, and the index of time slots is denoted by $h \in\{1,2, \ldots, H\}$. In the community $n$, the index of customers is denoted by $q \in\left\{1,2, \ldots, Q_{n}\right\}$. Notations are summarized in Table I.

\section{A. Community-Level Model}

The community-level model used in this paper adopts the one from our previous work [25]. For completeness, we include some details as follows. In the community $n$, the total monetary cost is defined as a convex function of the total load at each time slot in order to discourage the accumulation of energy load in any single time slot. There are various pricing models in existing literature, including the quadratic pricing model in [10], the linear pricing model in [1], the piecewise convex pricing model in [26], and the piecewise linear pricing model provided by British Columbia Hydro Corporation [10]. The quadratic function $C_{h, n}=a_{h, n} L_{h, n}^{2}$ is adopted in this paper
TABLE I

LIST OF NOTATIONS

\begin{tabular}{|c|c|}
\hline Variable Name & Variable Defination \\
\hline$a_{n, h}$ & The pricing parameter in community $n$ at time slot $h$ \\
\hline$L_{h, n}$ & The total energy load in community $n$ at time slot $h$ \\
\hline$C_{h, n}$ & The Total monetary cost of community $n$ at time slot $h$ \\
\hline$l_{q, h}$ & The energy consumption of customer $q$ at time slot $h$ \\
\hline$t s_{i}$ & Starting time of home appliance $i$ \\
\hline$t e_{i}$ & Ending time of home appliance $i$ \\
\hline $\mathcal{A}_{q}$ & Pome appliance set of customer $q$ \\
\hline $\mathcal{E}_{i}$ & Power level of home appliance $i$ at time slot $h$ \\
\hline$x_{i}^{h}$ & Total revenue of utility $m$ at time slot $h$ \\
\hline$w_{i}$ & Forward limit of utility $m$ at time slot $h$ \\
\hline$R_{m}^{h}$ & Reference profit of utility $m$ at time slot $h$ \\
\hline$d_{m, h}^{r e f}$ & Local buying cost of utility $m$ at time slot $h$ \\
\hline$b_{m, h}^{r e f}$ & Pricing parameter of utility $m$ at time slot $h$ \\
\hline$p_{m}^{h}$ & Energy selling of utility $m$ at time slot $h$ \\
\hline$a_{m}^{h}$ & Energy purchase of aggregator $n$ from utility $m$ at \\
\hline$d_{, m}^{h}$ & time slot $h$ \\
\hline$d_{n, m}^{h}$ & Energy demand of aggregator $n$ at time slot $h$ \\
\hline$d_{n, \cdot}$ & \\
\hline & \\
\hline
\end{tabular}

[10]. At the time slot $h, C_{h, n}$ is the total monetary cost in the community $n, L_{h, n}$ is the total energy load, and $a_{h, n}$ is the unit energy price, which converts energy consumption to monetary cost. Among the customers in this community, the expense is shared based on the individual energy consumption such that if the energy consumption of the customer $q$ is $l_{q, h}$ at the time slot $h$, the monetary cost is $C_{h, n} l_{q, h} / L_{h, n}$.

For the customer $q$, denote the set of home appliances by $\mathcal{A}_{q}$. For home appliance $i \in \mathcal{A}_{q}$, denote the set of power levels by $\mathcal{E}_{i}$. At the time slot $h$, the home appliance $i \in \mathcal{A}_{q}$ works in the power level $x_{i}^{h} \in \mathcal{E}_{i}$, which denotes the energy consumption in a single time slot. The home appliance $i$ is operated between the earliest start time $\mathrm{ts}_{i}$ and the latest end time te $\mathrm{e}_{i}$, whereas the required energy consumption is $w_{i}$. Since each customer aims to minimize its individual monetary cost, the optimization problem of customer $q$ in community $n$ is formulated as [25]

$$
\begin{gathered}
\min \sum_{h=0}^{H} \frac{C_{h, n} l_{q, h}}{L_{h, n}} \\
\text { s.t. } \sum_{h=\mathrm{ts}_{i}}^{\mathrm{te}_{i}} x_{i}^{h} \geq w_{i} \\
\sum_{i \in \mathcal{A}_{q}} x_{i}^{h}=l_{q, h} \\
\sum_{q=1}^{Q_{n}} l_{q, h}=L_{h, n} \\
x_{i}^{h} \in \mathcal{E}_{i} .
\end{gathered}
$$

\section{B. Market-Level Model}

The market competition is modeled here. At each time slot, the aggregators purchase electricity energy from the utilities based on the energy demand of the customers.

1) Market Operation of Utility: The selling pricing of utilities is based on the buying price from the generators. For this reason, the revenue of the utilities could be divided into two parts, including the estimated cost and the expected profit. For the utility $m$, when the amount of energy sold is equal to the 


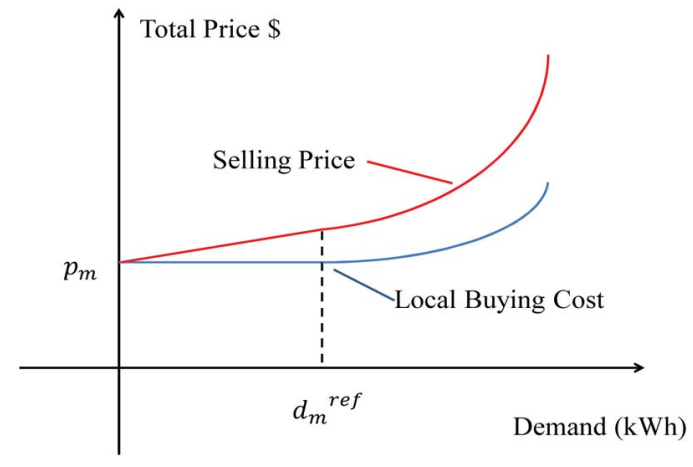

Fig. 5. Revenue and local buying cost of utility $m$ versus energy demand corresponding to (2).

forward limit $d_{m, h}^{\text {ref }}$, it is expected to make a profit as much as $b_{m, h}^{\mathrm{ref}}$, which is called the reference profit. As shown in Fig. 5, $p_{m}^{h}$ is the buying cost on the forward contract, and $a_{m}^{h}\left(d_{\cdot, m}^{h}\right)^{2}$ is the estimated buying cost when the amount of purchased energy exceeds the forward limit. Given the total energy demand $d_{r, m}^{h}$, the pricing function of utility $m$ is defined as

$$
R_{m}^{h}= \begin{cases}p_{m}^{h}+\frac{d_{,, m}^{h} b_{m, h}^{\mathrm{ref}}}{d_{m, h}^{\mathrm{ref}}}, & d_{\cdot, m}^{h} \leq d_{m, h}^{\mathrm{ref}} \\ a_{m}^{h}\left(d_{\cdot, m}^{h}\right)^{2}+\frac{d_{\cdot, m}^{h} b_{m, h}^{\mathrm{ref}}}{d_{m, h}^{\mathrm{ref}}}, & d_{\cdot, m}^{h}>d_{m, h}^{\mathrm{ref}} .\end{cases}
$$

In order to make $R_{m}^{h}$ a continuous function of $d_{,, m}^{h}$, set $p_{m}^{h}=$ $a_{m}^{h}\left(d_{m, h}^{\mathrm{ref}}\right)^{2}$. When energy demand $d_{\cdot, m}^{h} \leq d_{m, h}^{\mathrm{ref}}$, the utility $m$ could purchase this amount of energy in the forward market and sell it to the aggregators. The buying cost for the utility $m$ to keep the forward contract is $p_{m}^{h}$ with the expected profit of $d_{\cdot, m}^{h} b_{m, h}^{\mathrm{ref}} / d_{m, h}^{\mathrm{ref}}$. When $d_{\cdot, m}^{h}>d_{m, h}^{\mathrm{ref}}$, the utility $m$ estimates the buying cost as $a_{m}^{h}\left(d_{r, m}^{h}\right)^{2}$, which is the generation cost of the local generator. The expected profit is $d_{\cdot, m}^{h} b_{m, h}^{\mathrm{ref}} / d_{m, h}^{\mathrm{ref}}$.

While the buying price from the generators within the forward limit is always $p_{m}^{h}$, beyond the forward limit, the trading between utilities and generators is operated based on the LMP with discrete bidding as discussed in [24]. Each generator posts a price table based on the incremental price. A generator needs to satisfy the total request of the local utility before supplying the request of utilities in remote areas. The details are provided in [24, Ch. 4.3.1.5].

2) Market Operation of Aggregator: In the market level, aggregators purchase electricity energy from the utilities to meet the energy demand of their communities. The amount of energy purchased by aggregator $n$ from utility $m$ is denoted by $d_{n, m}^{h}$ such that $d_{,, m}^{h}=\sum_{n=1}^{N} d_{n, m}^{h}$. Similar to within a community, if multiple aggregators purchase from utility $m$, they share the total expense proportionally based on the trading amount with $m$. The total demand of an aggregator is denoted by $d_{n,}^{h}$, where $d_{n, .}^{h}=\sum_{m=1}^{M} d_{n, m}^{h}$. With the aforementioned definitions, the monetary cost minimization problem is formulated as

$$
\begin{gathered}
\min \sum_{m=1}^{M} \frac{d_{n, m}^{h}}{\sum_{n=1}^{N} d_{n, m}^{h}} R_{m}^{h} \\
\text { s.t. } \sum_{m=1}^{M} d_{n, m}^{h}=d_{n, \cdot \cdot}^{h} .
\end{gathered}
$$

\section{Game Formulation}

Here, the competitions in the community and market levels are modeled as a bi-level game. At the community level, each customer competes to reduce its individual monetary cost through selecting the power levels. At the market level, each aggregator competes to reduce the total monetary cost of its community through planning purchasing from the utilities. The complete game model is formulated as follows.

Bi-Level Game Model

\section{Community Level:}

1) Players: Customers.

2) Payoff functions: $-\sum_{h=0}^{H} \frac{C_{h, n} l_{k, h}}{L_{h, n}}$

3) Optimization problem:

$$
\begin{gathered}
\min \sum_{h=0}^{H} \frac{C_{h, n} l_{q, h}}{L_{h, n}} \\
\text { s.t. } \sum_{h=\mathrm{ts}_{i}}^{\mathrm{te}_{i}} x_{i}^{h}=w_{i} \\
\sum_{i \in \mathcal{A}_{q}}^{Q_{n}} x_{i}^{h}=l_{q, h} \\
\sum_{q=1}^{Q_{n}} l_{q, h}=L_{h, n} \\
x_{i}^{h} \in \mathcal{E}_{i}
\end{gathered}
$$

where $C_{h, n}=a_{h, n} L_{h, n}^{2}$

4) Decision Variables: $x_{i}$

\section{Market Level:}

1) Players: Aggregators

2) Payoff functions: $-\sum_{m=1}^{M}\left(d_{n, m}^{h} / \sum_{n=1}^{N} d_{n, m}^{h}\right) R_{m}$

3) Optimization problem:

$$
\begin{aligned}
& \min \sum_{m=1}^{M} \frac{d_{n, m}^{h}}{\sum_{n=1}^{N} d_{n, m}^{h}} R_{m} \\
& \text { s.t. } \sum_{m=1}^{M} d_{n, m}^{h}=d_{n, .}^{h} .
\end{aligned}
$$

where

$$
R_{m}^{h}= \begin{cases}p_{m}^{h}+\frac{d_{\cdot, m}^{h} b_{m, h}^{\mathrm{ref}}}{d_{m, h}^{\mathrm{ref}}}, & d_{\cdot, m}^{h} \leq d_{m, h}^{\mathrm{ref}} \\ a_{m}^{h}\left(d_{\cdot, m}^{h}\right)^{2}+\frac{d_{\cdot, m}^{h} b_{m, h}^{\mathrm{ref}}}{d_{m, h}^{\mathrm{ref}}}, & d_{\cdot, m}^{h}>d_{m, h}^{\mathrm{ref}}\end{cases}
$$

4) Decision Variables: $d_{n, m}^{h}$

As mentioned in Section II-A, each customer aims to reduce the electricity bill through assigning the working power levels of the home appliances given the electricity price in the community level. Note that the scheduling of each customer impacts the electricity bills of each other. This naturally leads to the competition between them modeled by the communitylevel game. Similarly, each aggregator determines the energy purchasing from each utility to minimize the total monetary 


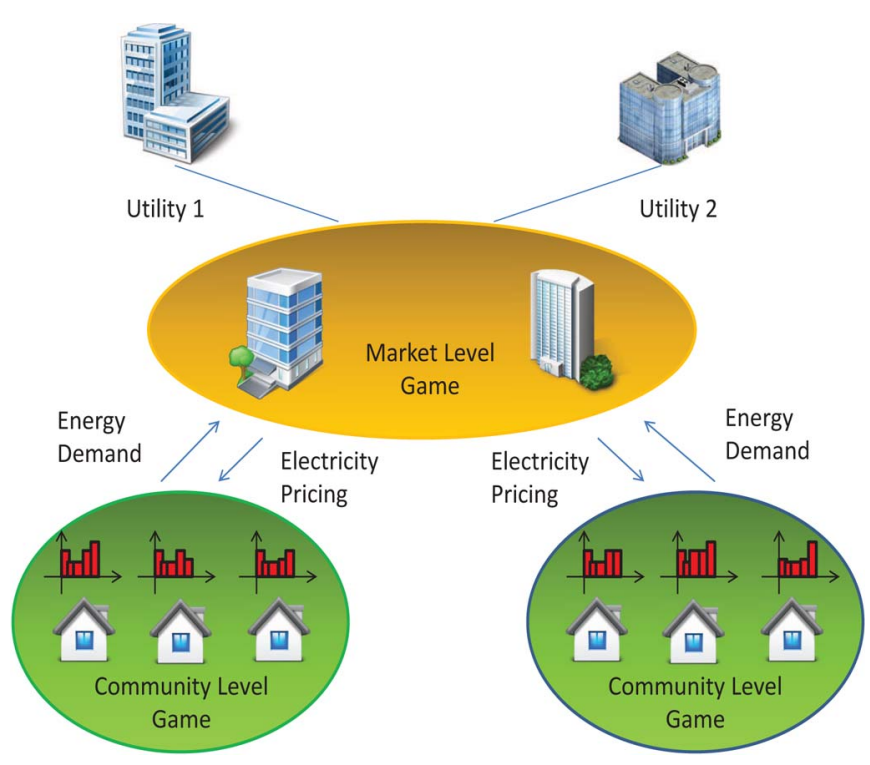

Fig. 6. Bi-level game model.

cost of the local community. It is formulated as a game since the purchasing of each aggregator impacts each other as well.

The two levels interact based on the electricity pricing and energy demand. The electricity pricing in each community is determined by the aggregator resulting from the competition of the market-level game. In return, the market-level game is based on the real-time energy demand in each community, which is the result of the competition in the community-level game. For each level, a pure strategy Nash equilibrium exists. The Nash equilibrium of the community-level game has been proved by several existing works, including [10] and [27]. In this paper, we prove the existence of Nash equilibrium of our market-level game in two steps. In the first step, we show that there exists a global optimal solution of $d_{n, m}^{h}$, which minimizes the total monetary cost of all the aggregators. In the second step, we show a closed-form solution of the pure strategy Nash equilibrium for our problem.

1) Step 1: There exists a global optimal solution of $d_{n, m}^{h}$ that minimizes the total monetary cost of all the aggregators.

In our formulation, the aggregators determine the energy purchasing $d_{n, m}^{h}$ to minimize the individual monetary cost. Given the energy demand of each community at each time slot $h$, the monetary cost of each aggregator $m$ is $\sum_{m=1}^{M}\left(d_{n, m}^{h} / \sum_{n=1}^{N} d_{n, m}^{h}\right) R_{m}$. The total monetary cost of all the aggregators is calculated as

$$
\sum_{n=1}^{N} \sum_{m=1}^{M} \frac{d_{n, m}^{h}}{\sum_{n=1}^{N} d_{n, m}^{h}} R_{m}=\sum_{m=1}^{M} R_{m},
$$

while $d_{n, m}^{h}$ is constrained by

$$
\sum_{m=1}^{M} d_{n, m}^{h}=d_{n, \cdot}^{h}
$$

Note that $R_{m}$ is a convex function of $d_{n, m}^{h}$, the linear combination of multiple such functions is also convex. Since the constraint conditions are linear, there exists a solution of $d_{n, m}^{h}$ that minimizes the total monetary cost $\sum_{m=1}^{M} R_{m}$ due to the convexity of the problem.

2) Step 2: A closed-form solution of the pure strategy Nash equilibrium is shown for the aforementioned problem.

In order to provide a closed-form solution, we solve the market-level game based on the assumptions as follows for simplicity.

a) The forward limit is set as $d_{m, h}^{\text {ref }}=0$. Without loss of generality, we can handle the problem similarly when $d_{m, h}^{\mathrm{ref}} \neq 0$.

b) At time slot $h$, the two aggregators have the same energy demand that $d_{1, \cdot}^{h}=d_{2, .}^{h}=\cdots=d_{N, \cdot}$.

Based on the aforementioned assumptions, each aggregator aims to minimize the individual monetary cost. However, one can show that the solution of problem

$$
\begin{aligned}
& \min \sum_{m=1}^{M} R_{m} \\
& \text { s.t. } \sum_{m=1}^{M} d_{n, m}^{h}=d_{n}^{h},
\end{aligned}
$$

is a Nash equilibrium of the market-level game. In order to simplify the analytical presentation, assume $N=2$ and $M=2$. However, the solution can be generalized to any $N$ and $M$. Using Lagrangian relaxation, the problem (6) is rewritten as

$$
\begin{aligned}
\min & a_{1}^{h}\left(d_{1,1}^{h}+d_{2,1}^{h}\right)^{2}+a_{2}^{h}\left(d_{1,2}^{h}+d_{2,2}^{h}\right)^{2} \\
& +\lambda_{1}\left(d_{1, \cdot}^{h}-d_{1,1}^{h}-d_{1,2}^{h}\right)+\lambda_{2}\left(d_{2, \cdot}^{h}-d_{2,1}^{h}-d_{2,2}^{h}\right) .
\end{aligned}
$$

It is easy to derive

$$
\begin{aligned}
& d_{1,1}^{h}+d_{2,1}^{h}=\frac{a_{2}\left(d_{1, \cdot}^{h}+d_{2, \cdot}^{h}\right)}{a_{1}^{h}+a_{2}^{h}} \\
& d_{1,2}^{h}+d_{2,2}^{h}=\frac{a_{1}\left(d_{1, \cdot}^{h}+d_{2, \cdot}^{h}\right)}{a_{1}^{h}+a_{2}^{h}} \\
& d_{1,1}^{h}+d_{1,2}^{h}=d_{1, \cdot}^{h} \\
& d_{2,1}^{h}+d_{2,2}^{h}=d_{2, \cdot}^{h} .
\end{aligned}
$$

Thus, the energy purchasing $d_{n, m}^{h}$ can minimize the total monetary cost as long as the constraints (8) are satisfied. A possible solution is

$$
\begin{aligned}
& d_{1,1}^{h}=d_{2,1}^{h}=\frac{a_{2}\left(d_{1, \cdot}^{h}+d_{2, \cdot}^{h}\right)}{2\left(a_{1}^{h}+a_{2}^{h}\right)} \\
& d_{1,2}^{h}=d_{2,2}^{h}=\frac{a_{1}\left(d_{1, \cdot}^{h}+d_{2, \cdot}^{h}\right)}{2\left(a_{1}^{h}+a_{2}^{h}\right)} .
\end{aligned}
$$

With this solution, the individual monetary cost of each aggregator is also minimized. Suppose that aggregator 1 aims to change the purchasing amount $d_{1,1}^{h}$ and $d_{1,2}^{h}$ to 
$d_{1,1}^{h}+\Delta d$ and $d_{1,2}^{h}-\Delta d$, respectively. Thus, the individual monetary costs before and after making this change are

$$
\begin{aligned}
\operatorname{Cost}_{1}= & a_{1}^{h}\left(d_{1,1}^{h}+d_{2,1}^{h}\right) d_{1,1}^{h}+a_{2}^{h}\left(d_{1,2}^{h}+d_{2,2}^{h}\right) d_{1,2}^{h} \\
\operatorname{Cost}_{1}^{\prime}= & a_{1}^{h}\left(d_{1,1}^{h}+d_{2,1}^{h}+\Delta d\right)\left(d_{1,1}^{h}+\Delta d\right) \\
& +a_{2}^{h}\left(d_{1,2}^{h}+d_{2,2}^{h}-\Delta d\right)\left(d_{1,2}^{h}-\Delta d\right)
\end{aligned}
$$

respectively.

Through changing the energy purchase by $\Delta d$, the individual monetary cost is increased by

$$
\begin{aligned}
\operatorname{Cost}_{1}^{\prime}-\operatorname{Cost}_{1}= & \left(a_{1}^{h}+a_{2}^{h}\right) \Delta d^{2}+\Delta d\left(a_{1}^{h} d_{2,1}^{h}-a_{2}^{h} d_{2,2}^{h}\right) \\
& +2 \Delta d\left(a_{1}^{h} d_{1,1}^{h}-a_{2}^{h} d_{1,2}^{h}\right) \\
= & \left(a_{1}^{h}+a_{2}^{h}\right) \Delta d^{2}>0 .
\end{aligned}
$$

Thus, each aggregator cannot unilaterally decrease the individual monetary cost in this situation. Thus, $\left(d_{1,1}^{h}, d_{1,2}^{h}, d_{2,1}^{h}, d_{2,2}^{h}\right)$ is a pure strategy Nash equilibrium of the market-level game. One can similarly extend this proof for any value of $N$ and $M$.

\section{Community-Level Algorithm}

At the community level, the customers schedule their home appliances in order to minimize their individual monetary costs. Given the electricity price, each customer solves the optimization problem (1) to set power levels and operation time of each home appliance. The dynamic programming algorithms proposed in our previous work [25] are used to solve the smart home scheduling problems among multiple customers.

\section{E. Market-Level Algorithm}

At the market level, each aggregator purchases electricity energy from the utilities in order to minimize the monetary cost and satisfy the total demand of its community. Since the total selling price of a utility is based on the total energy demand, the trading amount of one aggregator with a utility influences the monetary cost of the other aggregators. A distributed market purchasing algorithm is proposed to provide the solution of purchasing for each aggregator. With the feedback from the utilities, the aggregator $n$ computes $d_{n, m}^{h}$ that minimizes the monetary cost of its own community assuming the purchasing of the other aggregators is fixed. This is repeated until there is no change in the monetary costs of the aggregators. For each utility, the pricing function $R_{m}$ is a piecewise convex function such that it is a quadratic function when the total energy demand exceeds the forward limit, and it is linear otherwise. This makes the objective function of each aggregator more complex. To handle the optimization problem of aggregators flexibly, an energy demand partition technique is used in the market purchasing algorithm.

The energy demand partition-based market purchasing algorithm is given in Algorithm 1. Within each iteration, the aggregator $n$ divides the total demand $d_{n}^{h}$. into $K_{n}$ pieces, where each piece is $d_{n,}^{h} / K_{n}$. The aggregator $n$ maintains $\left[d_{n, 1}^{h}, d_{n, 2}^{h}, \ldots, d_{n, M}^{h}\right]$ as the decision array. Each time, the aggregator chooses a utility to purchase the energy piece $k \in\left\{1,2, \ldots, K_{n}\right\}$ to minimize the current monetary cost. Assuming that the aggregator $n$ purchases the $k$ th piece of energy from utility $m$, the pricing of utility $m$ is changed according to (2). Thus, the aggregator $n$ chooses the current cheapest utility to purchase the $(k+1)$ th piece of energy. This is repeated until the total amount of energy demand is placed. A complete description of the algorithm is presented in Algorithm 1. In order to implement the aforementioned procedure, we introduce a bidding array $\left[d_{n, 1}, d_{n, 2}, \ldots, d_{n, M}\right]$ and temporary monetary cost $\left[\mathrm{tc}_{1}, \mathrm{tc}_{2}, \ldots, \mathrm{tc}_{M}\right]$ as temporary variables.

The algorithm initializes in lines 1 and 2 . In line 7 , the aggregator places the energy piece $k$ to each utility and obtains the corresponding temporary monetary cost $\mathrm{tc}_{m}$ in line 8 . In line 11 , the aggregator chooses the utility $\hat{m}$ with the minimum temporary monetary cost to purchase the energy piece $k$. Subsequently, the decision array is updated in line 12 .

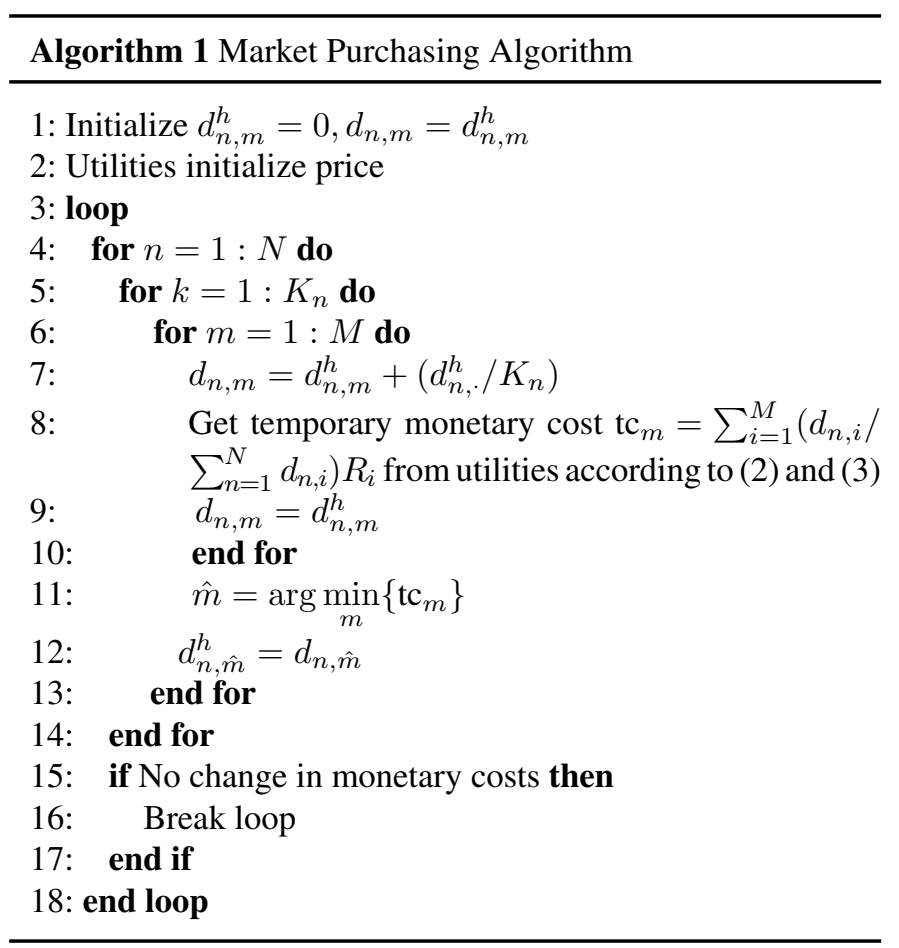

\section{F. Top-Level Algorithm}

While the algorithms are provided to solve the smart home scheduling problem and the market purchasing problem, respectively, the bidirectional influence between the levels should be considered. The electricity price is needed for the communities to conduct smart home scheduling, and the energy demand is needed for the trading operation in the market. A top-level algorithm is proposed to link smart home scheduling and market purchasing together, which is given in Algorithm 2. In line $1, a_{h, n}$ is initialized. From line 3 to line 5, the dynamic programming algorithm in [25] is called to solve the smart home scheduling problem under the current pricing. In line 6, Algorithm 1 is called to solve the market purchasing problem. Since the aggregators can only obtain the total monetary cost from the market, it is converted into unit price in line 7. When the energy load of community $n$ is $L_{h, n}$ and the total monetary cost is $C_{h, n}$ at time slot $h, a_{h, n}$ is updated as 


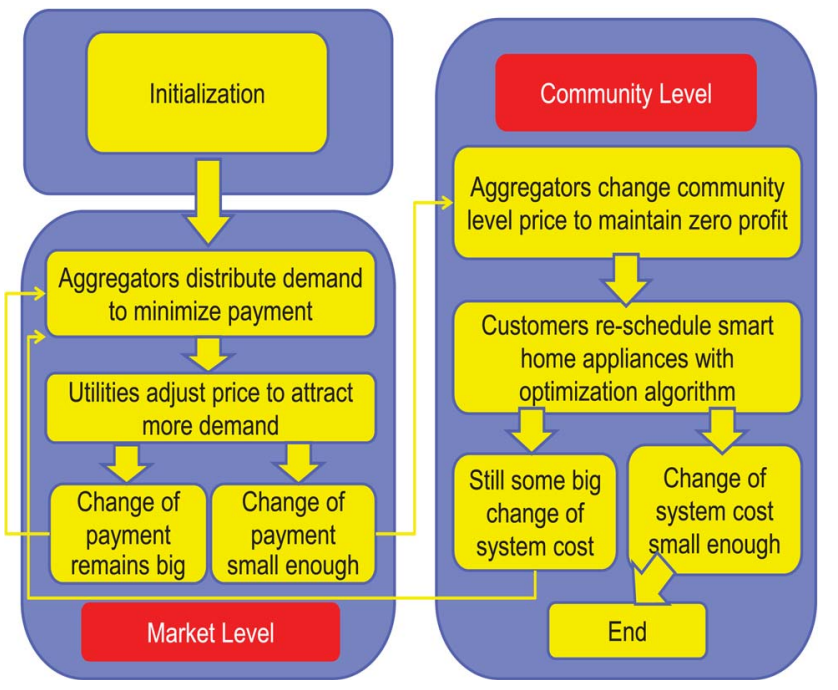

Fig. 7. Proposed algorithm consists of the smart home scheduling algorithm in the community level and the market purchasing algorithm in the market level. The two levels are linked together as a loop to emulate the bidirectional influence of each other.

$a_{h, n}=C_{h, n} / L_{h, n}^{2}$, which is to be used in the next iteration. The complete algorithm flow is shown in Fig. 7.

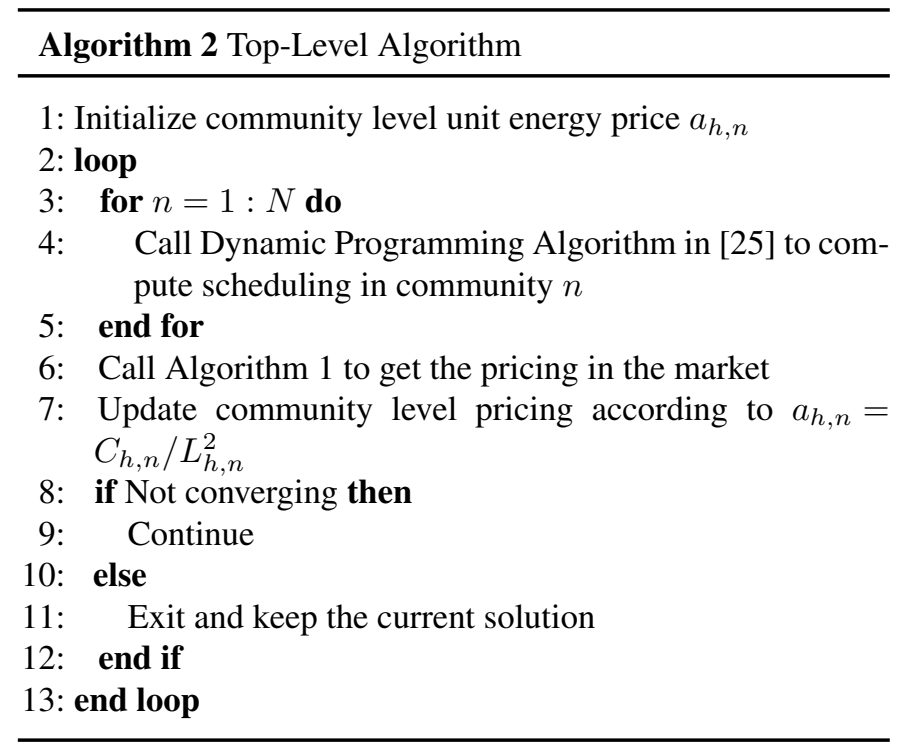

\section{CAse Study}

Here, simulations are conducted, and the impact of smart homes to the electricity market is analyzed.

\section{A. Simulation Setup}

In our generated benchmark, there are two utilities and five communities, where each community consists of 400 customers. Each customer is equipped with both automatically controlled home appliances and manually controlled home appliances. The daily energy consumption and execution duration of automatically controlled home appliances are given in Table II, which is obtained from the data provided by [3], [10], [28], and [29]. In the simulation, the execution period and energy consumption are randomly chosen from the corresponding
TABLE II

DAILY ENERGY CONSUMPTION AND RUN TIME OF AUTOMATICALLY Controlled Home ApPliances

\begin{tabular}{|c|c|c|}
\hline Home Appliance & Daily Consumption & Execution Duration \\
\hline Washing Machine & $1.2 \mathrm{kWh}-2 \mathrm{kWh}$ & $0.5 \mathrm{~h}-1.5 \mathrm{~h}$ \\
\hline Dish Washer & $1.2 \mathrm{kWh}-2 \mathrm{kWh}$ & $0.5 \mathrm{~h}-1 \mathrm{~h}$ \\
\hline Cloth Dryer & $1.5 \mathrm{kWh}-3 \mathrm{kWh}$ & $0.5 \mathrm{~h}-1.5 \mathrm{~h}$ \\
\hline PHEV & $9 \mathrm{kWh}-12 \mathrm{kWh}$ & $4 \mathrm{~h}-8 \mathrm{~h}$ \\
\hline Air Conditioner & $2 \mathrm{kWh}-3 \mathrm{kWh}$ & $1 \mathrm{~h}-3 \mathrm{~h}$ \\
\hline Heater & $2 \mathrm{kWh}-3 \mathrm{kWh}$ & $1 \mathrm{~h}-3 \mathrm{~h}$ \\
\hline
\end{tabular}
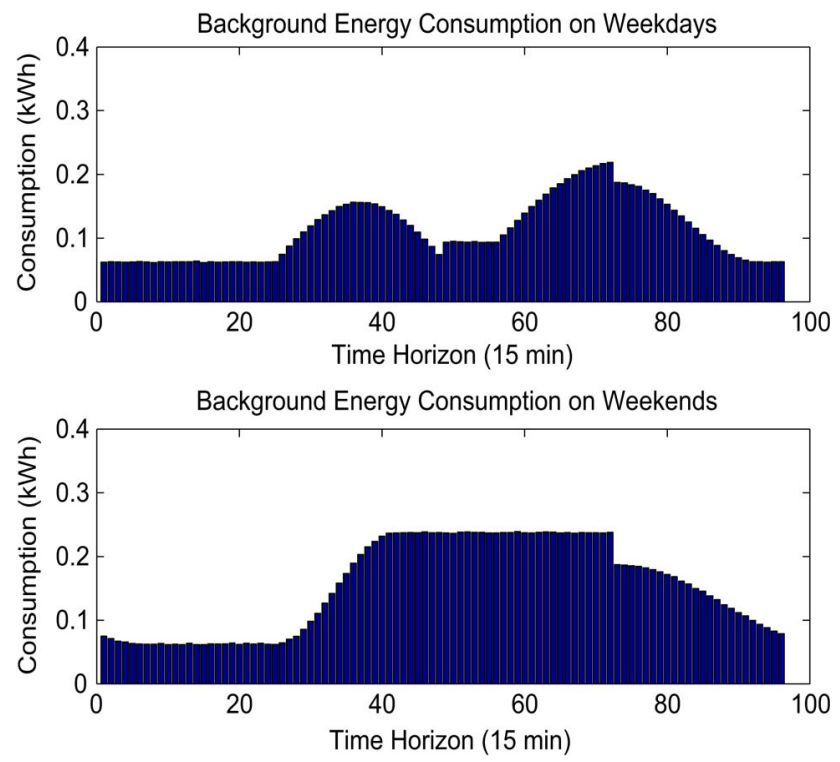

Fig. 8. Background consumption on weekdays and weekends, which are created by the manually controlled home appliances.

ranges provided in the table with a uniform distribution. For each customer, the energy consumption of the manually controlled home appliances is fixed, whereas the energy consumption of automatically controlled home appliances is scheduled by the smart scheduling algorithm. Since the energy consumption profile created by the manually controlled home appliances is not changeable, it is called background energy consumption.

Two different scenarios, including weekdays and weekends, are considered in the benchmark. Generally speaking, people use the home appliances more on weekends than weekdays. For this reason, in the simulation, more home appliances are operated on weekends, and the execution durations are longer than weekdays. Fig. 8 depicts the average background energy consumption of one customer on weekdays and weekends, respectively. They are generated according to [3], [28], and [30]. The peak energy consumption appears from evening to night when most people are off work at home. There is also a lower peak energy consumption during the daytime around the noon. The energy consumption starts to increase from the morning and reaches the peak around the noon.

For each utility, the parameters in (2) are set according to the following rules. 1) The purchasing cost $p_{m}$ within the forward limit is set according to the average energy demand at each time slot and the generation cost. 2) The purchasing price $a_{m}^{h}$ beyond the forward limit is set according to the generation cost. For utility 1 , the parameters are set as $p_{1}^{h}=\$ 30, b_{1, h}^{\mathrm{ref}}=\$ 10$, and 

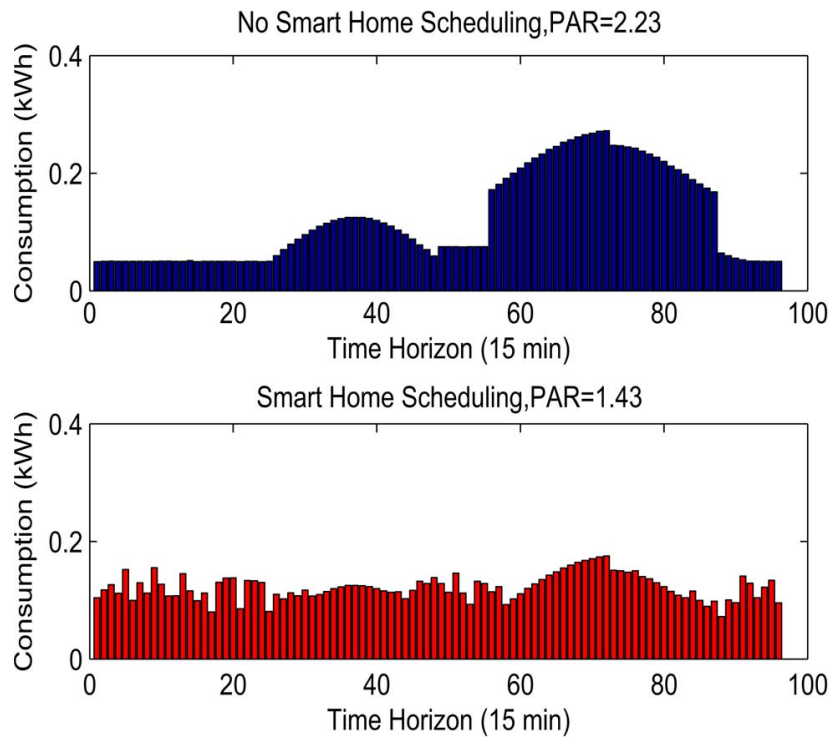

Fig. 9. Average energy consumption of a customer on weekdays.
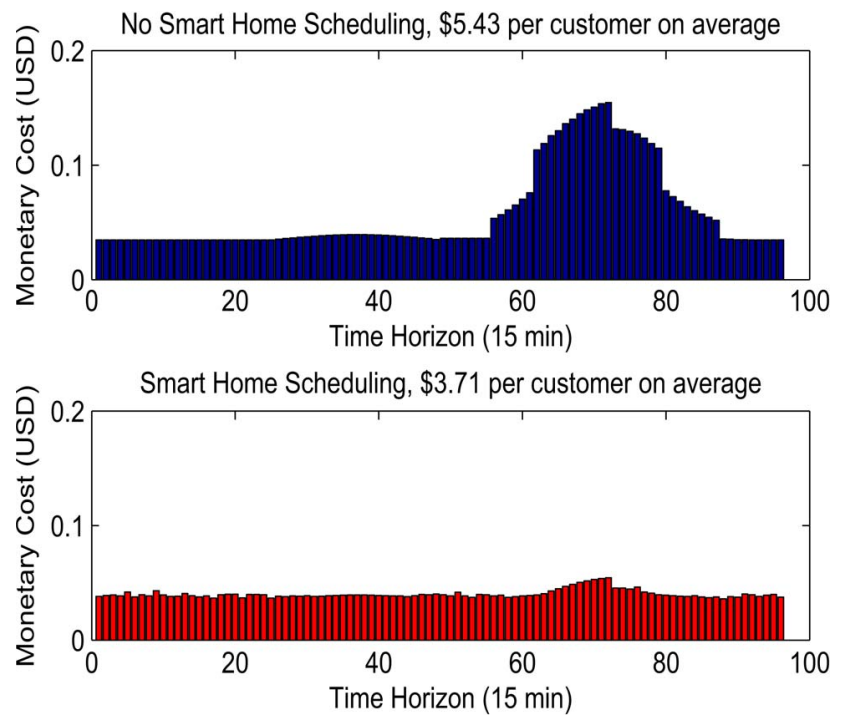

Fig. 10. Average monetary cost of each customer on weekdays.

$a_{1}^{h}=0.12 \$ / \mathrm{kWh}^{2}$. For utility 2 , the parameters are set as $p_{2}^{h}=$ $\$ 33, b_{2, h}^{\text {ref }}=\$ 10$, and $a_{2}^{h}=0.13 \$ / \mathrm{kWh}^{2}$. The scheduling time is $24 \mathrm{~h}$ from $12 \mathrm{~A} . \mathrm{M}$. of the current day to $12 \mathrm{~A} . \mathrm{M}$. next day, which is divided into 15 -min time slots.

The simulation results for weekdays are presented as follows. The average energy consumption profile for a customer is shown in Fig. 9. With smart home scheduling, the energy consumption is balanced over the time horizon. This reduces the PAR by $35.9 \%$, from 2.23 to 1.43 , which is also the reduction rate of the peak of the total generation requirement. Note that the monetary cost at a time slot will be even higher as the energy consumption accumulates. When smart home scheduling is applied, the energy consumption of the automatically controlled home appliances is scheduled at the time slots without heavy background load. Thus, the energy load is shifted off the peak, which results in balanced energy usage.

As shown in Fig. 10, the average monetary cost of each customer is also evenly distributed due to the balancing of
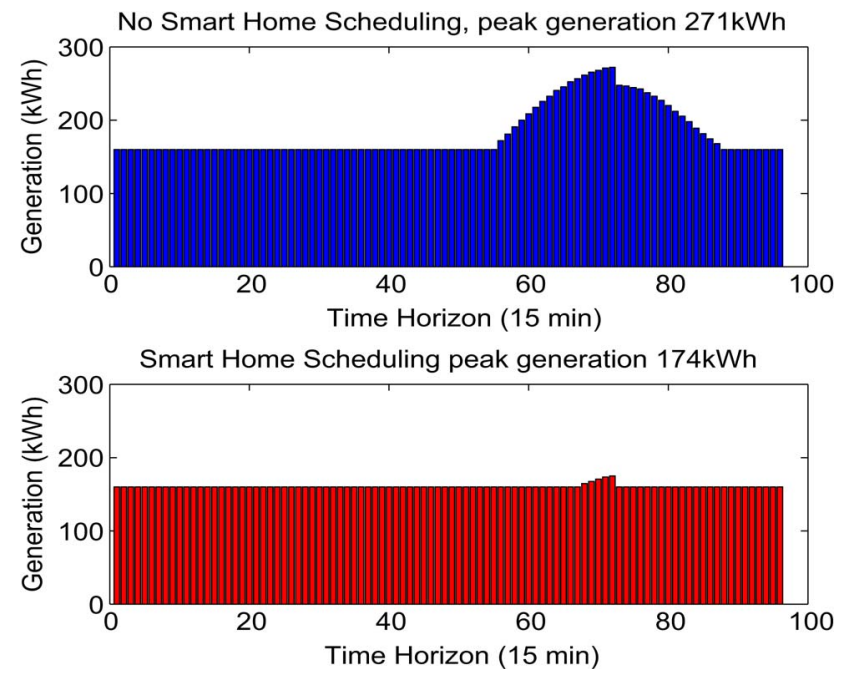

Fig. 11. Energy generation of generator 1 on weekdays.
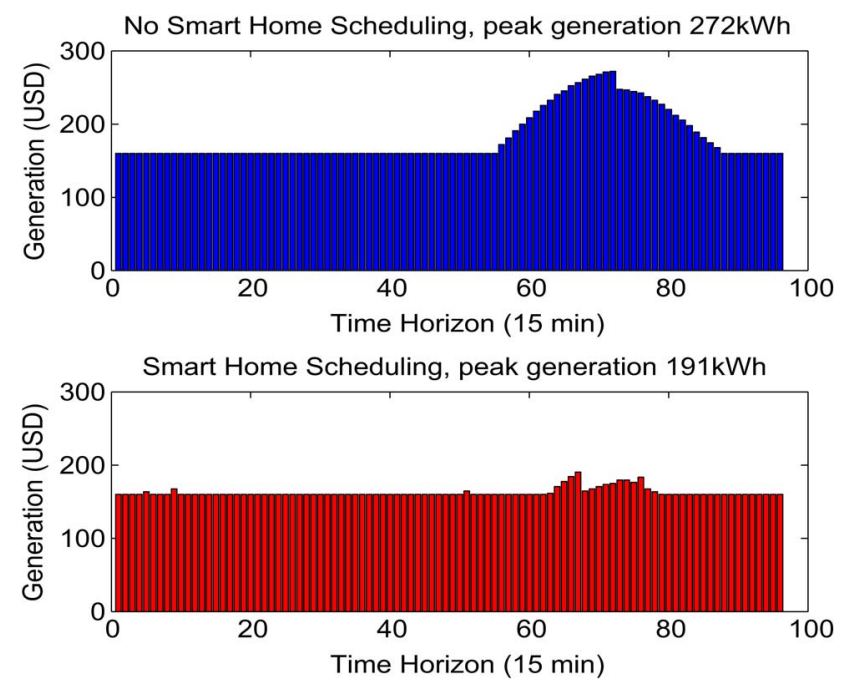

Fig. 12. Energy generation of generator 2 on weekdays.

the energy consumption. The monetary cost of all the customers over the whole time horizon is reduced by $31.8 \%$, from $\$ 5.43$ to $\$ 3.71$. Since the total energy cost is independent of smart home scheduling, the reduction of total monetary is due to the mitigation of the high purchasing price in the market.

The energy generation of the two generators over the time horizon are shown in Figs. 11 and 12. The generated energy is balanced among all time slots, which demonstrates that smart home scheduling can implicitly help balance the energy generation. As obtained from Figs. 11 and 12, the peak generation of generator 1 is reduced by $36.0 \%$, from 271 to $174 \mathrm{kWh}$, and the peak generation of generator 2 is reduced by $29.8 \%$, from 272 to $191 \mathrm{kWh}$.

Our iterative algorithm is effective in balancing the energy consumption from iteration to iteration. As an example, the energy consumption of community 1 in four consecutive iterations is summarized in Fig. 13, which clearly shows that the energy consumption becomes more and more balanced during the optimization. 

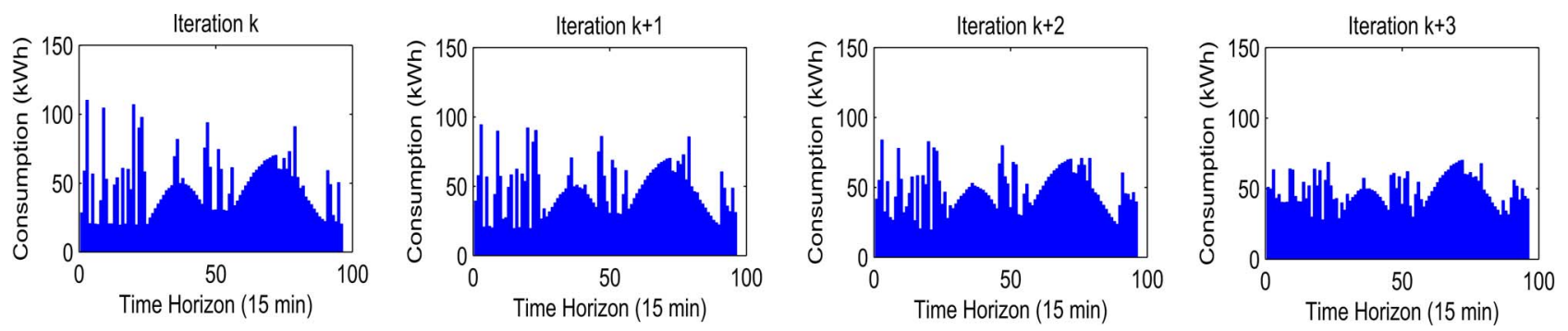

Fig. 13. Total energy consumption of community 1 in four consecutive iterations.
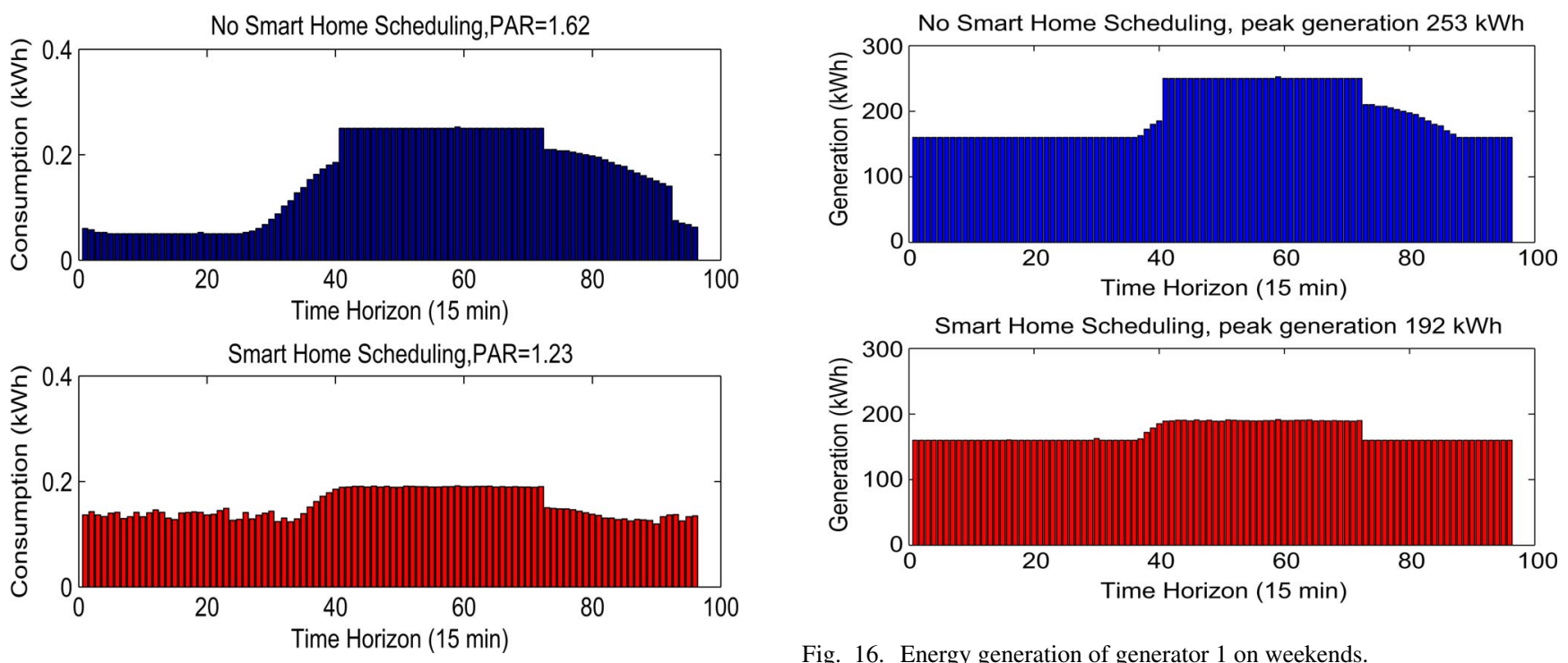

Fig. 14. Average energy consumption of the customers on weekends.

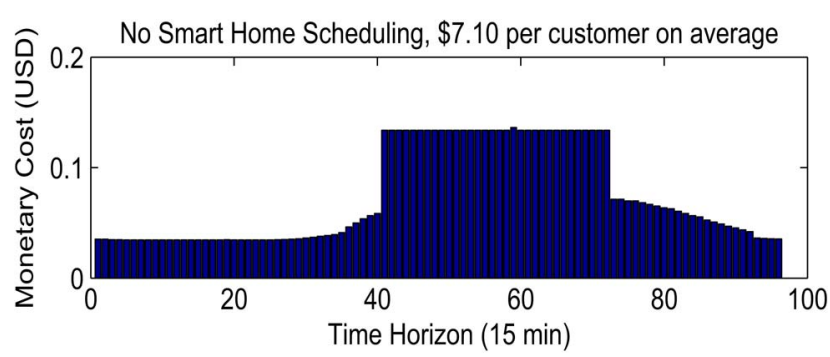

Fig. 16. Energy generation of generator 1 on weekends.
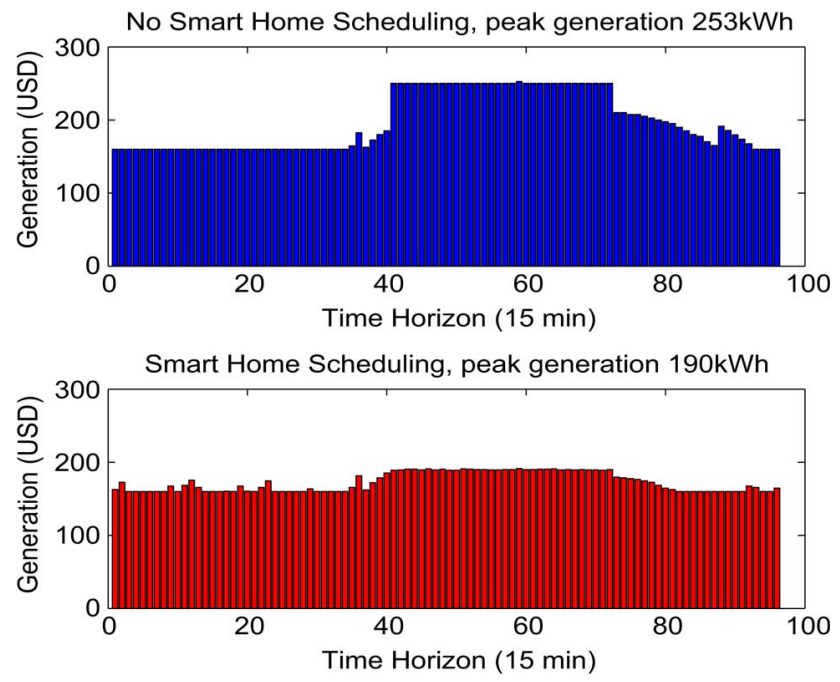

Fig. 17. Energy generation of generator 2 on weekends.

Fig. 15. Average monetary cost of each customer on weekends.

\section{B. Results for Weekends}

Fig. 14 shows the average energy consumption of each customer with and without smart home scheduling, respectively. Similar to the result for weekdays, smart home scheduling helps balance the energy load. However, comparing with Fig. 9, the total energy consumption is increased because the home appliances are operated longer. On weekends, the PAR is 1.23 with smart home scheduling, whereas it is 1.62 without smart home

scheduling, which is a $24.9 \%$ reduction. As shown in Fig. 15, on weekends, the total monetary cost of customer is reduced by $34.3 \%$, from $\$ 7.10$ to $\$ 4.66$, by smart home scheduling.

The energy generation of the two generators on weekends is shown in Figs. 16 and 17. Similar to the results on weekdays, the generation is more balanced resulting from smart home scheduling. For generator 1, the peak generation is reduced by $24.1 \%$, from 253 to $192 \mathrm{kWh}$. For generator 2, the peak generation is reduced by $24.9 \%$, from 253 to $190 \mathrm{kWh}$. 


\section{CONCLUSION}

In a smart community infrastructure that consists of multiple smart homes, smart controllers schedule various home appliances to balance energy consumption and reduce the electricity bills of customers. This paper analyzes the impact of the smart home scheduling to the electricity market. It also proposes a new smart-home-driven bi-level market model where the customers schedule home appliances for bill reduction at the community level, whereas aggregators minimize the energy purchasing expense from utilities at the market level, both of which consider the smart home scheduling impacts. A gametheoretic algorithm is proposed to solve this formulation, which handles the bidirectional influence between the community level and the market level. As demonstrated by simulation results, the average monetary cost of customers is reduced by $31.8 \%$ on weekdays and by $34.3 \%$ on weekends. In addition, the PAR for the energy consumption is reduced by $35.9 \%$ on weekdays and by $24.9 \%$ on weekends. Furthermore, the peak generation requirement can be reduced by $29.8 \%$ on weekdays and by $24.9 \%$ on weekends.

\section{REFERENCES}

[1] X. Chen, T. Wei, and S. Hu, "Uncertainty-aware household appliance scheduling considering dynamic electricity pricing in smart home," IEEE Trans. Smart Grid, vol. 4, no. 2, pp. 932-941, Jun. 2013.

[2] A. Mohsenian-Rad and A. Leon-Garcia, "Optimal residential load control with price prediction in real-time electricity pricing environments," IEEE Trans. Smart Grid, vol. 1, no. 2, pp. 120-133, Sep. 2010.

[3] [Online]. Available: http://www.eia.gov/

[4] [Online]. Available: http://www.powersmartpricing.org/

[5] T. Kim and H. Poor, "Scheduling power consumption with price uncertainty," IEEE Trans. Smart Grid, vol. 2, no. 3, pp. 519-527, Sep. 2011.

[6] L. Liu, X. Yang, H. Huang, and S. Hu, "Smart home scheduling for cost reduction and its implementation on FPGA," J. Circuit, Syst. Comput., vol. 24, no. 4, pp. 1-15, 2015.

[7] K. Sou, J. Weimer, H. Sandberg, and K. Johansson, "Scheduling smart home appliances using mixed integer linear programming," in Proc. IEEE CDC-ECC, 2011, pp. 5144-5149.

[8] K. Tsui and S. Chan, "Demand response optimization for smart home scheduling under real-time pricing," IEEE Trans. Smart Grid, vol. 3, no. 4, pp. 1812-1821, Dec. 2012.

[9] A. Mohsenian-Rad, V. Wong, J. Jatskevich, and R. Schober, "Optimal and autonomous incentive-based energy consumption scheduling algorithm for smart grid," in Proc. ISGT, 2010, pp. 1-6.

[10] A. Mohsenian-Rad, V. Wong, J. Jatskevich, R. Schober, and A. Leon-Garcia, "Autonomous demand-side management based on game-theoretic energy consumption scheduling for the future smart grid," IEEE Trans. Smart Grid, vol. 1, no. 3, pp. 320-331, Dec. 2010.

[11] C. Ibars, M. Navarro, and L. Giupponi, "Distributed demand management in smart grid with a congestion game," in Proc. IEEE Int. Conf. SmartGridComm, 2010, pp. 495-500.

[12] C. Wu, A. Mohsenian-Rad, and J. Huang, "Vehicle-to-aggregator interac tion game," IEEE Trans. Smart Grid, vol. 3, no. 1, pp. 434-442, Mar. 2012.

[13] S. Kishore and L. Snyder, "An innovative RTP-based residential power scheduling scheme for smart grids," in Proc. IEEE ICASSP, 2011, pp. 5956-5959.

[14] S. Bu, F. Yu, and P. Liu, "Dynamic pricing for demand-side management in the smart grid," in Proc. IEEE Online Conf. GreenCom, 2011, pp. 47-51.

[15] T. Aigner, H. Farahmand, and T. Gjengedal, "Modeling the northern European electricity market," in Proc. IEEE Power Energy Soc. Gen. Meet., 2012, pp. 1-8.

[16] G. Knezevic, K. Fekete, and S. Nikolovski, "Applying agent-based modeling to electricity market simulation," in Proc. Int. Conv. Inf. Commun. Technol. Electron. MIPRO, 2010, pp. 647-652.

[17] M. Hassan, M. Abdullah, A. Arifin, F. Hussin, and M. Majid, "Electricity market models in restructured electricity supply industry," in Proc. IEEE Int. PECon, 2008, pp. 1038-1042.
[18] E. Bompard, Y. Ma, R. Napoli, G. Gross, and T. Guler, "Comparative analysis of game theory models for assessing the performances of network constrained electricity markets," IET Gener., Transmiss. Distrib., vol. 4, no. 3, pp. 386-399, 2010.

[19] J. Zhang, J. Fuller, and S. Elhedhli, "A stochastic programming model for a day-ahead electricity market with real-time reserve shortage pricing," IEEE Trans. Power Syst., vol. 25, no. 2, pp. 703-713, May 2010.

[20] T. Voice, P. Vytelingum, S. D. Ramchurn, A. Rogers, and N. R. Jennings, "Decentralised control of micro-storage in the smart grid." in Proc. AAAI Conf. Artif. Intell., 2011, pp. 1-7.

[21] S. D. Ramchurn, P. Vytelingum, A. Rogers, and N. R. Jennings, "Agentbased homeostatic control for green energy in the smart grid," ACM Trans. Intell. Syst. Technol., vol. 2, no. 4, p. 35, Jul. 2011.

[22] K. KoK et al., "Smart houses for a smart grid," in Proc. Int. Conf. Exhib. Elect. Distrib., Part 1, 2009, pp. 1-4.

[23] X. Guan, J. Wu, F. Gao, and G. Sun, "Optimization-based generation asset allocation for forward and spot markets," IEEE Trans. Power Syst., vol. 23, no. 4, pp. 1796-1808, Nov. 2008.

[24] D. Kirschen and G. Strbac, Fundamentals of Power System Economics. Hoboken, NJ, USA: Wiley, 2004.

[25] L. Liu, Y. Zhou, Y. Liu, and S. Hu, "Dynamic programming based game theoretic algorithm for economical multi-user smart home scheduling," in Proc. IEEE Int. MWSCAS, 2014, pp. 362-365.

[26] S. Caron and G. Kesidis, "Incentive-based energy consumption scheduling algorithms for the smart grid," in Proc. IEEE Int. Conf. SmartGridComm, Oct 2010, pp. 391-396.

[27] C. Wu, H. Mohsenian-Rad, J. Huang, and A. Wang, "Demand side management for wind power integration in microgrid using dynamic potential game theory," in Proc. IEEE GC Wkshps, 2011, pp. 1199-1204.

[28] [Online]. Available: http://www.absak.com/library/power-consumptiontable

[29] K. Herter, P. McAuliffe, and A. Rosenfeld, "Observed temperature effects on hourly residential electric load reduction in response to an experimental critical peak pricing tariff," U.S. Dept Energy, Ernest Orlando Lawrence Berkeley Nat. Lab., Berkeley, CA, USA, 2005.

[30] P. Asare et al., "Real time pricing of electric power," School Elect. Eng., Purdue University, West Lafayette, IN, USA, 1995.

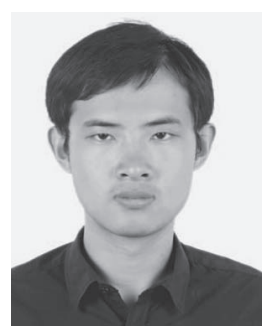

Yang Liu received the B.S. degree from the Huazhong University of Science and Technology, Wuhan, China, in 2011. He is currently working toward the $\mathrm{Ph} . \mathrm{D}$. degree in electrical engineering at Michigan Technological University, Houghton, MI, USA.

His research focuses on smart home systems, cyber-physical systems, and big data analysis.

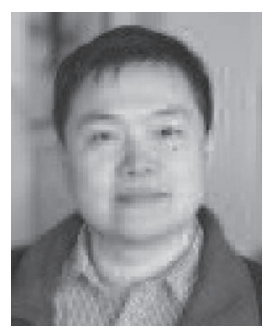

Shiyan Hu (SM'10) received the Ph.D. degree in computer engineering from Texas A\&M University, College Station, TX, USA, in 2008.

$\mathrm{He}$ is currently an Associate Professor with the Department of Electrical and Computer Engineering, Michigan Technological University, Houghton, MI, USA. He was a Visiting Professor at IBM Research (Austin) during summer of 2010. His research interests are in the area of computer-aided design of VLSI circuits, cyber-physical systems, and smart home cybersecurity, where he has published over 80 technical papers in refereed journals and conferences.

Dr. Hu has served as General Chair, Technical Program Committee (TPC) Chair, TPC Subcommittee Chair, Session Chair, and TPC Member for various conferences for more than 70 times. He is an Associate Editor or Guest Editor of the IEEE TRANSACTIONS ON CIRCUITS AND SySTEMS-PART II, IEEE TRANSACTIONS ON COMPUTERS, IEEE TRANSACTIONS ON COMPUTERAIDED DESIGN, IEEE TRANSACTIONS ON INDUSTRIAL INFORMATICS, and ACM Transactions on Embedded Computing Systems. He was a recipient of the ACM SIGDA Richard Newton DAC Scholarship (as the Faculty Advisor), the Faculty Invitation Fellowship from the Japan Society for the Promotion of Science, and the National Science Foundation CAREER Award. 


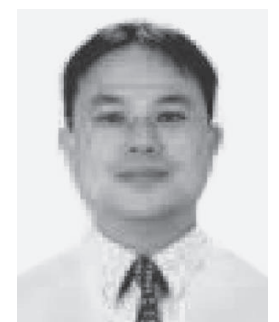

Han Huang (SM'08) received the Ph.D. degree from New York University Polytechnic School of Engineering, Brooklyn, NY, USA, in 2006.

He is currently the Deputy Director of the Smart Grid Research Department, State Grid Energy Research Institute, State Grid Corporation of China, Beijing, China. He has published various papers in books, journals, conferences, and workshops. His research interests include smart grids, power systems, and power electronics.

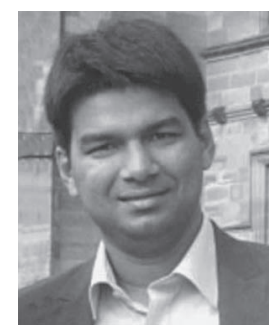

Rajiv Ranjan (M'08) received the Ph.D. degree in engineering from the University of Melbourne, Parkville, Australia, in 2009.

$\mathrm{He}$ is a Research Scientist and a Julius Fellow at the Computational Informatics Division, Commonwealth Scientific and Industrial Research Organisation (CSIRO; formerly known as CSIRO ICT Centre). He has published 62 scientific peer-reviewed papers ( 7 books, 25 journals, 25 conferences, and 5 book chapters). His expertise is in datacenter cloud comoptimization.

puting, application provisioning, and performance

Dr. Ranjan has been invited to serve as the Guest Editor for leading distributed systems journals, including the IEEE TRANSACTIONS ON CLOUD Computing, Future Generation Computer Systems, and Software-Practice and Experience. One of his papers was in 2011's top computer science journal, i.e., the IEEE COMMUNICATION SURVEYS AND TUTORIALS. His h-index is 20, with a lifetime citation count of more than 1660 (Google Scholar). His papers have also received more than 140 ISI citations. Seventy percent of his journal papers and $60 \%$ of conference papers have been A*/A ranked ERA publication.

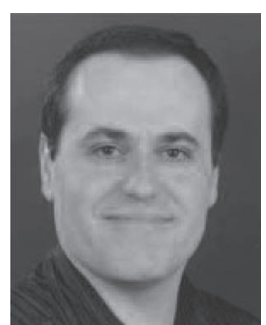

Albert Y. Zomaya (F'04) received the Ph.D. degree from the Department of Automatic Control and Systems Engineering, Sheffield University in Sheffield, U.K. He is currently the Chair Professor of High Performance Computing and Networking at the School of Information Technologies, The University of Sydney, Sydney, Australia. He is also the Director of the Centre for Distributed and High Performance Computing, which was established in late 2009. He has published more than 500 scientific papers and articles and is the author, coauthor, or editor of more than 20 books. His research interests are in the areas of parallel and distributed computing and complex systems.

Prof. Zomaya is a Chartered Engineer and a Fellow of the American Association for the Advancement of Science and The Institution of Engineering and Technology (U.K.). He served as the Editor in Chief of the IEEE TRANSACTIONS ON COMPUTERS (2011-2014). He currently serves as the Editorin-Chief of Springer's Scalable Computing and is an Associate Editor of 22 leading journals, such as the ACM Computing Surveys, IEEE TRANSACTIONS on COMPUTATIONAL Social Systems, IEEE TRANSACTIONS ON CLOUd Computing, and Journal of Parallel and Distributed Computing. He was a recipient of the IEEE Technical Committee on Parallel Processing Outstanding Service Award in 2011, the IEEE Technical Committee on Scalable Computing Medal for Excellence in Scalable Computing in 2011, and the IEEE Computer Society Technical Achievement Award in 2014.

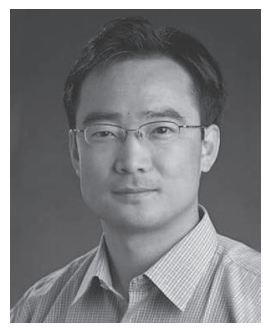

Lizhe Wang (SM'09) received the B.Eng. degree (with honors) and the M.Eng. degree from Tsinghua University, Beijing, China, and the Dr. Ing. degree (magna cum laude) in applied computer science from the University Karlsruhe (now Karlsruhe Institute of Technology), Karlsruhe, Germany.

$\mathrm{He}$ is a "100-Talent Program" Professor at the Institute of Remote Sensing and Digital Earth, Chinese Academy of Sciences, Beijing, and a "ChuTian" Chair Professor at the School of Computer Science, China University of Geosciences,

Wuhan, China.

Prof. Wang is a Fellow of The Institution of Engineering and Technology and the British Computer Society. 